\title{
Novel Detection Service Method for Moving Object in IOT
}

\author{
Jie Chen ${ }^{1, a}$, Degan Zhang ${ }^{2,3, b *}$ \\ ${ }^{1}$ School of Electronic and Information Engineering, Tianjin Vocational Institute, Tianjin, 300410, \\ China \\ ${ }^{2}$ Tianjin Key Lab of Intelligent Computing \& Novel software Technology, Tianjin University of \\ Technology, Tianjin, China \\ ${ }^{3}$ Key Laboratory of Computer Vision and System (Tianjin University of Technology),Ministry of \\ Education, 300384, China \\ aemail: xchenjie@hotmail.com, ${ }^{\mathrm{b} e m a i l: ~ g a n d e g a n d e @ 126 . c o m, ~ * C o r r e s p o n d e n c e ~ a u t h o r ~}$
}

Keywords: GMM; embedded; IOT; moving object; detection service

\begin{abstract}
In embedded Internet of Things (IOT) environment, there are the troubles such as complex background, illumination changes, shadows and other factors for detecting moving object, so we put forward a new detection service method through mixing Gaussian Mixture Model (GMM), edge detection service method and continuous frame difference method in this paper. In time domain, the new method uses GMM to model and updates the background. In spatial domain, it uses the hybrid detection service method which mixes edge detection service method, continuous frame difference method and GMM to get initial contour of moving object, and gets ultimate moving object. This method not only can well adapt to the illumination gradients and background disturbance occurred on scene, but also can well solve some problems such as inaccurate target detection, incomplete edge detection, cavitation and ghost which usually appears in traditional method. As experimental result showing, this method holds better real-time and robustness. It is not only easily implemented, but also can accurately detect moving object.
\end{abstract}

\section{Introduction}

As we know, in embedded Internet of Things (IOT) environment, vision is an important way for humans to observe and recognize the IOT, and the majority of the external information are obtained by vision. This not only shows the visual information occupies major share, but also reflects the importance of visual function in IOT environment. Human gives device of IOT a function like human vision. People use embedded camera to get images and convert them into digital signals in order to use embedded IOT device to process information, which is the process of IOT vision emergence [1-4]. The IVS (Intelligent Video Surveillance) in IOT achieves video surveillance intelligent process by using IOT vision technology to process, analyze and understand video signal so as to control the video monitoring system.

With the development of in embedded IOT technology, the IVS technology becomes important requirements. It uses open architecture structure, and easily integrates with alarm, access control, patrol, management information system. Based on embedded IOT technology, it has stable performance, good flexibility, and does not need the special management [6]. The IVS technology in embedded IOT environment mainly includes detecting and tracking moving object. So far, there are mainly three kind methods to test moving object in embedded IOT environment : Optical Flow method [7], inter-frame difference method [8] and background difference method [9]. The advantage of optical flow method is that it can detect the independence moving object, and needn't know any information about scene in advance.

\section{Principle of the basic methods}

The process of edge detection by using Canny operator mainly includes low pass filtering by Gaussian function, calculating the amplitude and direction of gradient, Non maximum suppression on the gradient amplitude, threshold and connected edge. 
1) Image low pass filtering

The Canny operator firstly uses first-order derivative of 2D Gaussian function to make low pass filter to image. The 2D Gaussian function is

$$
G(x, y)=\frac{1}{2 \pi \sigma^{2}} \exp \left(-\frac{x^{2}+y^{2}}{2 \sigma^{2}}\right)
$$

2) Calculate the amplitude and direction of gradient

Using Gauss function separability, we decompose the two filter faltung template of $\Delta G$ into two one-dimensional row and column filter, and then respectively calculate faltung with image $f(x, y)$. The result is

$$
\begin{gathered}
E_{x}=\frac{\partial G}{\partial x} * f(x, y), \quad E_{y}=\frac{\partial G}{\partial y} * f(x, y) \\
A(x, y)=\sqrt{E_{x}^{2}(x, y)+E_{y}^{2}(x, y)} \\
\partial(x, y)=\arctan \left[\frac{E_{y}(x, y)}{E_{x}(x, y)}\right]
\end{gathered}
$$

$A(x, y)$ and $\partial(x, y)$ respectively reflects gradient and direction angle size for $(x, y)$ of image.

3) Non maximum suppression on the gradient amplitude

It is insufficient to determine edge only by obtaining global gradient. So, we must retain the local maximum of gradient, and suppress the non maximum. The specific procedure is traversing the image. If the gradient amplitude value of a pixel is not biggest, through comparing with its front and behind pixel at the same gradient direction, we set this pixel value as 0 . That is to say, we retain only those points which have great local change at amplitude direction.

4) Threshold and edge connection

The non maximum suppression image is made two thresholds $T_{h}$ and $T_{l}$. They have the relationship $T_{h}=2 T_{l}$. Setting the gray level of a pixel as 0 , when the pixel's gradient value less than $T_{l}$, we will get the image $a$. Then setting those its gradient less than $T_{h}$ as 0 , we will get the image $b$. Due to the reason that the two image are set different threshold, while the image $b$ is wiped off most noise, some useful edge information also is deleted and the image $a$ preserves more information. We take the image $b$ as the basic image, and then use the image $a$ to supplement and connect the edge of image. Because the traditional Canny method has weak self-adaptability. We adopt the Otsu method to dynamically determine threshold, which can greatly improve the method self-adaptability.

The basic principle of inter-frame difference method is subtracting the corresponding pixel value of two neighboring frame image. In the case of environmental brightness change is not great, if the corresponding pixel value has little difference, we can think the scenery is motionless. If pixel values of some region in the scene have great changes, we reckon it is caused by target motion. Then marking the pixel which has great change, we can obtain the moving object by those marked pixel. The traditional adjacent frame method is easy to implement, calculating speed is fast, and having stronger robustness and self-adaption for dynamic environment. But it usually can't absolutely extract all relevant pixels, and it is easy to cause hollow in moving objects. Based on the shortcoming of the traditional inter-frame difference method, we design the continuous frame difference method. Many experiments have shown that it can overcome the shortcomings and deficiencies of traditional inter-frame difference method in a way.

The basic principle of continuous frame difference method is: firstly, we take $\mathrm{n}$ continuous frame images from a video image sequence. Then, in these continuous $n$ frame images, we make subtract operation which is respectively using the pixel of different frame image and the corresponding pixel of the middle image, and then make "AND" operation for those subtraction results. Finally, we get the result of moving object by adding the operation result. This method 
weakens the influence of environmental factors and noise on the detection of targets. In this paper, we will take continuous 7 frame image to calculate.

\section{Realization of the method}

The method combines the edge detection service method and the mixture Gauss mode. Firstly, we need modeling and updating the background during the time domain by using the mixture Gauss model, at the same time, testing the shadow by using the shadow model based on the RGB color space, and obtaining the initial shape of the moving objects. Then using the Canny edge detection service method to get the edge profile of the moving object in a spatial domain and calculate the results which obtaining from the above two steps with logical operation "AND”, so we can getting the edge profile of the moving objects.

Compared with the traditional detection service method about the moving objects, our method can solve the leakage phenomenon to a certain extent, which is due to the targets moving slowly in the scene. In some way, it also can make up for the problem about the incomplete detection of the edge in target domain. The specific description of the method in this paper is as follows.

1) Detect the contour of targets by using the method which combines mixture Gauss model with edge detection service method, specific steps of the mixture method are as follows:

(1) Model and update the background in the time domain by using the mixture Gauss model.

(2) With the foreground which is extracted by using the mixture Gauss model, detecting and eliminating the shadow in the RGB color space, thus we can extracted the initial foreground target.

(3) Detect the contour of the target by using the Canny edge detection service method.

2) Detect the contour of the target by using the inter-frame difference method and the edge detection service method, the specific steps of this mixture method are as follows:

(1) Randomly select 7 consecutive frames of video images from the surveillance video.

(2) Smoothing these 7 consecutive frames in the video image respectively, which selected from the surveillance video.

(3) Detect the images which have been smoothed by using the method of edge detection service method.

(4) Take the images with the inter frame difference, which have been detected by the edge detection service method, and make the threshold binarization.

3) To calculate the results which obtained from the step 1) and step 2) with "OR" operation, we can achieve the complete contour of the moving objects after the final confirmation.

4) Dealing the detected moving objects with morphology process, we can get the final detection targets.

For the moving foreground which detected by the mixture Gauss model, we need to make shadow suppression. In a scene, due to the light source is sheltered by the targets, and a shadow is produced, and the shadow moves along with the targets. The produced shadow will change the brightness of pixel in the background image, so it is obvious that both the shadow and the moving objects will be detected as a moving foreground. Removing the shadow is the key to improve the accuracy of detection. The view experience shows that in certain brightness the tone is equal whether the target is under the shadow or not. Tone is the index to divide the category of color and we take the RGB color space as an example. We proportionally reduce all of coefficients about certain color, and darken the brightness only, keep the tone unchanged. Therefore, the relationship of brightness between inside and outside the shadow area is as follows

$$
\begin{aligned}
Y_{s} & =0.299 R_{s}+0.587 G_{s}+0.114 B_{s} \\
& =0.299 K \times R+0.587 K \times G+0.114 K \times B \\
& =K \times(0.299 R+0.587 G+0.114 B)
\end{aligned}
$$

After the multi-color image is converted into the gray image, the pixel values when targets are under the shadow area is $K$ times to the corresponding value in the background model $(K<1)$, while the ratio of the brightness of foreground target with background is not a constant. Then, we can get the following formula under the illumination function 


$$
D(x, y)=\frac{F(x, y)}{B(x, y)}=\left\{\begin{array}{l}
1 \quad(x, y) \text { belongs to the background region } \\
k(x, y) \text { belongs tothe shadow region, } k \in(0,1) \\
\text { uncertain }(x, y) \text { belongs to the moving object } t \text { arg et }
\end{array}\right.
$$

This method can effectively reduce the results of the shadow effect on target detection, among them, $F(x, y)$ is real time image, $B(x, y)$ is background image, and $k=0.79$ in this paper.

After we get the binary images, it usually emerges some noise due to the effect of some factors, these noise is not beneficial to analysis and study about the target which we are interested in for further steps. In order to eliminate the noise in the binary images, we can deal the binary image with the morphological process to achieve this purpose.

Firstly, we remove the isolated noise point by the corrosion operation, and then, fill the edge hole of targets by using the dilation operation. Assuming that there is a function of binary image is $A(x, y)$, the template element is $B(x, y)$, and the corrosion is defined as follows:

$$
E=A \otimes B=\left\{x, y \mid B_{x y} \subseteq D\right\}
$$

That is to say, the binary image which produced by the template $B$ corroding the image $A$ is a set of points: If we move $B$ from the original point to $(x, y)$ and get $B_{x y}, B_{x y}$ will completely contain in $A$. Under the condition of computer implementation, we put the original point of $B(0,0)$ to a position in image $A$. If all of the points in $B$ are covered by image $A$, the point which the original of $B$ is the corresponding point that obtained from the corrosive results of $E$, otherwise this point is not belonged to the results of $E$. After traversing all the points in image $A$, the trajectory of original point of $B$ is the corresponding corrosive results.

And corresponding with the corrosion, assuming that there is a function of binary image is $A(x, y)$, the template element is $B(x, y)$, and the corrosion is defined as follows:

$$
D=A \oplus B=\left\{x, y\left|B_{x y}\right| A \neq \Phi\right\}
$$

That is to say, the binary image which produced by the template $B$ corroding the image $A$ is a set of points: If we move $B$ from original point to $(x, y)$ and get $B_{x y}$, the intersection that $B$ and $A$ is not empty. Under the condition of computer implementation, we put the original point of $B(0,0)$ into a position in image $A$. If both the points in $B$ and the corresponding points in $A$ are 1 , the point which the original of $B$ is the corresponding point which obtained from the expansible results of $D$, otherwise this point is not belonged to results of $D$. After traversing all points in image, trajectory of original point of B is corresponding expansible results.

\section{Comparisons of experimental result}

Simulation experiment is done in MATLAB. in order to verify the effectiveness of the proposed method in this paper, we test respectively a group of AVI video sequence image by edge detection service method, the mixed method including three frame difference method, five frame difference and background difference method, and the improved method in this paper. Making the pre-treatment for each method, we can get the detected results as shown in Figure 1, 2.

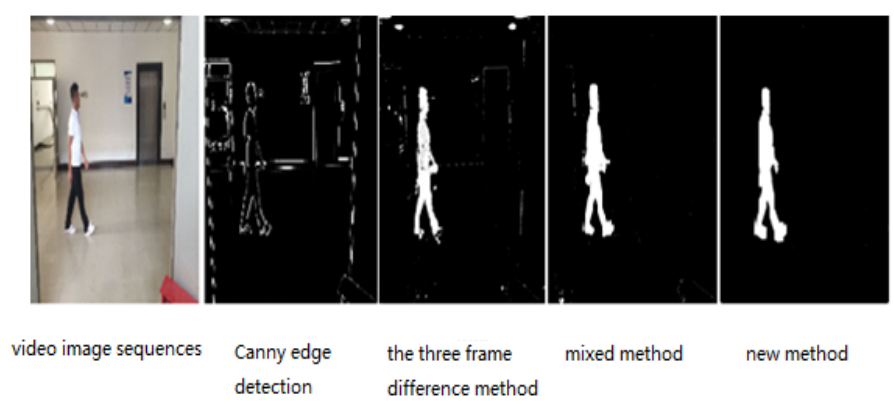

Figure 1 Comparison 1 of the detected result 


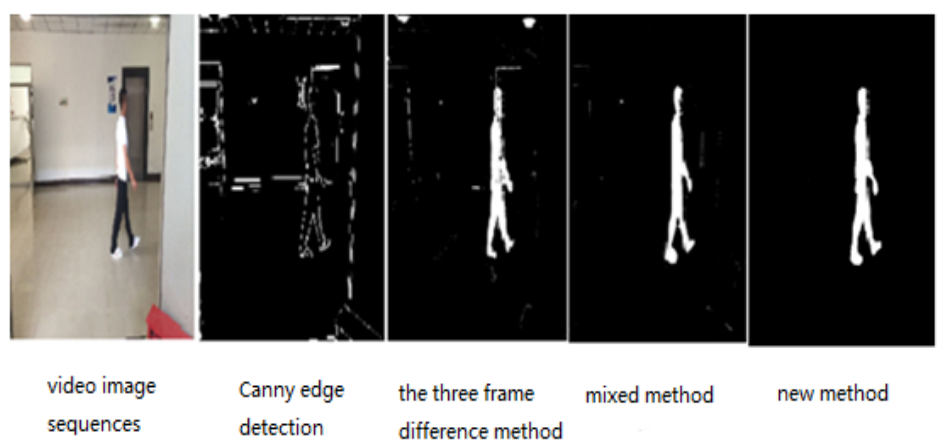

Figure 2 Comparison 2 of the detected result

As for a set of image in a video sequence, we use the method proposed in this paper and a mixed method which includes the three frame difference method, the five frame difference and the background difference method to handle image. The processing results as Figure 1 to Figure 2 are shown. The traditional three frame difference method can detect the moving object, but the target contour is not complete. The mixed method of five frame difference method and background subtraction method can improve the continuity and integrity of target contour in a way. The target detection service method in this paper can further enhance the completeness of the moving object, and in large part, eliminate target cavitation. It makes the detection results become accurate, complete, clear, and can satisfy the requirement of real-time detection.

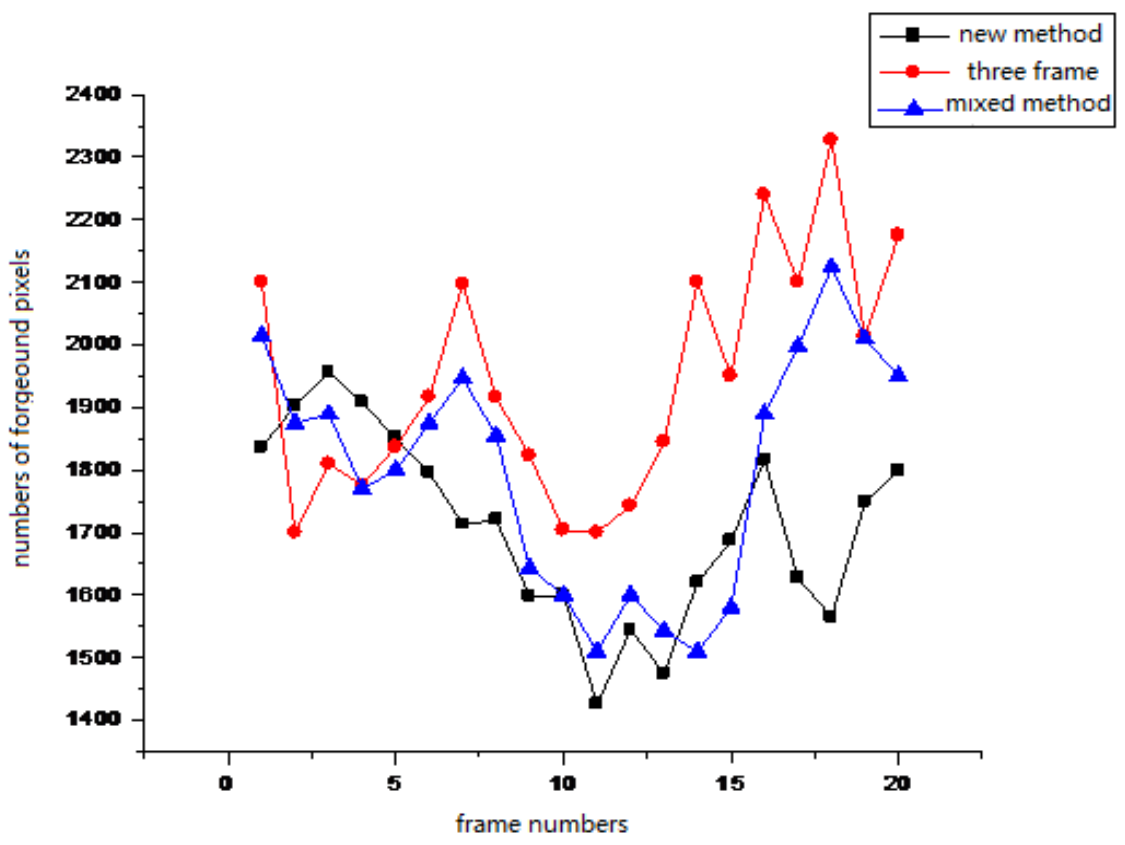

Figure 3 Comparison on pixel of three kinds of methods

As you can see from Figure 3, the number of foreground pixel which is obtained by the three frame difference method and the mixed method including the five frame difference and the background difference method is larger than that number of pixel which is obtained by the method proposed in this paper. This is mainly because the two methods have higher error rate through detecting the background pixel to foreground pixel than the method in this paper. The superiority of the method in this paper can be seen from the above quantitative contrast. In addition, we contrast the correct rate, aiming at different method of different scene at the same time range, and also contrast the complexity of the three methods, such as the following table 1 . The table 1 shows that in different scene the rate accuracy of the method in this paper is obviously higher than the other two methods. The complexity of the method in this paper decides the detection time of each frame is longer than the two methods. It also is the aspect which needs us to deeply study and improve. 
Table 1 Comparison on average time per frame detection

\begin{tabular}{|c|c|c|c|}
\hline $\begin{array}{l}\text { Average time per frame } \\
\text { detection } \\
\text { scene }\end{array}$ & $\begin{array}{l}\text { Three frame } \\
\text { difference }\end{array}$ & $\begin{array}{l}\text { Five frame difference \& } \\
\text { background } \\
\text { method combining method }\end{array}$ & $\begin{array}{l}\text { The method } \\
\text { proposed in this } \\
\text { paper }\end{array}$ \\
\hline Corridor interior & 0.279 & 0.405 & 0.575 \\
\hline Stopping place & 0.296 & 0.397 & 0.597 \\
\hline Store entrance & 0.301 & 0.436 & 0.612 \\
\hline Hostel entrance & 0.314 & 0.425 & 0.635 \\
\hline Gymnasium interior & 0.299 & 0.417 & 0.623 \\
\hline
\end{tabular}

\section{Conclusions}

We have proposed a new detection service method based on the mixture Gauss model, the inter-frame difference method and the edge detection service method for detecting the moving object in embedded IOT environment. This new method makes full use of the advantages of these three methods effectively, detects the contour of the moving objects in the same scene by using the mixture methods which is different from each other, and then takes the contours of targets calculated by these two different methods with "OR" operation. And it can not only confirm the edge information of the detected moving object but also complete the information, conquer some issues of the traditional methods that due to detecting the target incompletely, the shadow and the missing detect caused by the targets moving slowly.

\section{Acknowledgement}

This research work is supported by National Natural Science Foundation of China (Grant No. 61571328), Major projects of science and technology in Tianjin (No.15ZXDSGX00050), Training plan of Tianjin University Innovation Team (No.TD12-5016).

\section{References}

[1] Zhang D G, Kang X J. A novel image de-noising method based on spherical coordinates system, EURASIP Journal on Advances in Signal Processing,2012,2012(110):1-10

[2] Zhang D G, Li G. An energy-balanced routing method based on forward-aware factor for Wireless Sensor Network. IEEE Transactions on Industrial Informatics, 2014,10(1):766-773

[3] Zhang D G, Wang X. A Novel Approach to Mapped Correlation of ID for RFID Anti-collision. IEEE Transactions on Services Computing, 2014,7(4):741-748

[4] Zhang D G, Zhang X D. Design and implementation of embedded un-interruptible power supply system (EUPSS) for web-based mobile application. Enterprise Information Systems, 2012,6(4):473-489.

[5] Zhang D G, Zhu Y N. A new constructing approach for a weighted topology of wireless sensor networks based on local-world theory for the Internet of Things (IOT). Computers \& Mathematics with Applications, 2012,64(5):1044-1055

[6] Zhang D G, Liang Y P. A kind of novel method of service-aware computing for uncertain mobile applications. Mathematical and Computer Modelling, 2013,57(3-4):344-356.

[7] Zhang D G, Zheng K, Ting Zhang. A Novel Multicast Routing Method with Minimum Transmission for WSN of Cloud Computing Service. Soft Computing, 2015,19(7):1817-1827.

[8] Zhang D G. A new approach and system for attentive mobile learning based on seamless migration. Applied Intelligence, 2012, 36(1):75-89 
[9] Zhang D G, Song X D. New Agent-based Proactive Migration Method and System for Big Data Environment (BDE). Engineering Computations,2015,32(8):2443-2466 